in appraising and interpreting evidence type in the hierarchy of evidence-based medicine resource's pyramid. EBM curriculum will include longitudinal training both in inpatient and outpatient setting to capture all relevant point of care patient centered clinical queries mapped to specialty topics of insufficient evidence. A built-in knowledge resource, multi modal teaching methods including small group teaching, e- learning and journal club will ensure a comprehensive training in EBM. The ultimate outcome will be a certified training in evidence-based medicine at the conclusion of the residency training for life long evidence-based practice.

Results We have implemented a web-based EBM curriculum platform hosted at www.ebmcentral.net. Preliminary testing at various workshops have been conducted with positive feedback. Beta study results showed that web based longitudinal curriculum provides an excellent opportunity to train students, resident and faculty in practicing evidence-based medicine through a formal longitudinal EBM curriculum. A clinically integrated curriculum along with innovative EBM tools provides a simulated article reading and appraisal training. A point of care query capturing tool and meta search engine for EBM resources provides a convenient platform to trigger selfdirected point of care learning for EBM cycle. Self-directed web-based assignments along with both summative and formative assessment ensure a continuous growth of required competencies in longitudinal curriculum timeline. Lastly, research dissemination through a collaborative crowd research environment to synthesize research evidence such as systematic review is also integrated.

Conclusions We have conceived and implemented a web based longitudinal curriculum for a certified training in evidencebased medicine i.e Certified evidence-based medicine practitioner (CEBMP). Our platform has provided preliminary supportive evidence of enhancing reflective learning, knowledge gaps and needs assessment, and clinically integrated formal training in evidence-based medicine. A personal knowledge repository along with acquired skills of evidence-based medicine including question formulation, information mastery, critical appraisal, research synthesis and interpretation provides a foundation for lifelong self-directed learning for evidencebased practice. We plan to conduct a randomized clinical trial to assess the role of our longitudinal curriculum in providing certified training in evidence-based medicine to medical students, residents and faculty.

\section{PATIENT AND PUBLIC INVOLVEMENT IN RESEARCH AGENDA-SETTING AND CLINICAL TRIALS: WHY, WHEN AND HOW}

Michele Kok. University of the West of England, Bristol, UK

\subsection{6/bmjebm-2019-EBMLive.42}

Objectives The research agenda in medicines development has traditionally been established without input from its beneficiaries i.e. patients. However, patient and public involvement (PPI) is increasingly being recognised as important in medicines development, to ensure more meaningful medicines development by the pharmaceutical industry and generate new insights to aid regulatory review and decision-making. Besides, involving the public in any decision that affects their health is ethical, helps create a culture of transparency, and improves acceptance of decisions. The increased relevance to patients may subsequently lead to improved recruitment and retention in clinical trials, another key stage in the medicine's development lifecycle. Other benefits of PPI in clinical trials include reduced numbers of protocol amendments and better accessibility of resulting evidence to the public. Given the lack of guidance on PPI in research agenda-setting and clinical trials, we aimed to develop practical evidence-based guidance on the role of PPI in these areas.

Method Development of the PPI guidance involved three main tasks. First, was a critical review of existing literature and resources to collate material related to PPI in research agendasetting and clinical trials. The next task involved an iterative process, working collaboratively with the PPI Panel for Antimicrobial Drugs (PPIPAD), comprising individuals who had experienced an acute infection requiring admission into an intensive-care unit, either personally or as a carer. At each PPIPAD meeting, members were presented with a draft version of the guidance, and asked to comment on aspects such as language, layout and content. Researchers carefully considered these contributions and revised the guidance accordingly. This process was repeated until a final version was produced and agreed. Lastly, this co-produced guidance was piloted at a workshop for project managers and officers at the Julius Centre, University Medical Centre Utrecht, renowned for its applied clinical research and innovations in clinical research methodology.

Results The guidance presents evidence for the role of PPI, including practical 'how-tos' and real-world examples. It describes two approaches to PPI in research agenda-setting: the Dialogue Model, and the James Lind Alliance Priority Setting Partnerships. In clinical trials, patients (different from patients participating in a trial) can be involved in trial design and protocol development by advising on the relevance of patient-reported outcomes and outcome measures, improving access to and recruitment of participants, and assisting in the development of patient-related materials (informed consent documents, data collection tools). In trial conduct, patients have a role in supporting operations and clinical infrastructure, advising on any trial adaptations, and ensuring accountability of researchers. The role of PPI in trial data analysis and dissemination of results includes ensuring coherence in the understanding and interpretation of data, contributing to the analysis of participant feedback on trial experiences, and assisting in the development of patientlevel communication.

Conclusions There is potential to incorporate PPI from an early stage in the medicines development lifecycle i.e. setting the research agenda, and across all stages of the clinical trial continuum. In addition to the benefits of PPI, we acknowledge the challenges associated with it. Successful implementation of PPI in these areas requires clear goals and welldeveloped plans for responsive and managerial PPI roles, equal partnership between stakeholders and patients, and the provision of ongoing information and education to empower and facilitate the active involvement of patients.

This guidance for PPI in research agenda-setting and clinical trials has been included as two individual chapters in the PPI Toolkit and Practical Guide for Antimicrobial Medicines Development Research, developed for COMBACTE-MAGNET's clinical coordinating work package. COMBACTE-MAGNET (Combatting Bacterial Resistance in Europe - Molecules against Gram-Negative Infections) is a consortium of academic and industry researchers committed to seeking new ways to treat multi-resistant bacterial infections. 